\title{
Organizaciones no gubernamentales y sociedad civil en El Salvador
}

\author{
Oscar Morales Velado
}

\section{Iniroducción}

El presente estudio, representa uno de varios esfuerzos personales por investigar la dinámica e impaclo social de los procesos de afirmación de la sociedades civiles en Centroamérica y particularmenle en El Salvador, frente a la secular dominación de las sociedades políticas. En estos procesos han surgido y se han redelinido nuevos actores, entre los cuales se cuentan a las organizaciones no gubernamentales, las cuales desempefian un papel importanle en la creación de una nueva sociedad civil y en la construcción del consenso nacional.

El concepto de organización no gubernamental es aclualmente discutible, especialmente porque su identidad social se está formando por su accionar. Para fructíficar la discusión, debe admitirse como temas de la misma: Primero, los procesos de surgimiento de las ONG'S, en situaciones de guerra civil o posteriores a éstas y el segundo, alude tanto a las moditicaciones organizativas como a los campos de acción de esas instiluciones privadas, signilicalivamenle diterenles a otras experiencias.

Según Cuenca y confirmando lo anteriormente mencionado, las ONG'S, pertenecen a una parte de la sociedad civil y ésla es: " el conjunlo de organizaciones dirigidas o gerenciadas en el ámbito de lo privado o de la solidaridad ciudadana o gremial. Esta red organizacional que llamamos sociedad civil promueve diversas demandas e intereses de los grupos sociales, entre ellos: delensa económica, de género, de promoción humana y desarrollo, cientílicos y cullurales, entre otros. Distinguiremos a la sociedad civil del mundo inslilucional surgido y articulado estructural 
y funcionalmente al Eslado. También la dislinguiremos de la sociedad polílica, fundamenlalmente partidos y movimienlos políticos, cuya meta común es alcanzar el ejercicio del poder estatal. Finalmente la distinguiremos del nivel de lo social no organizado" (Cuenca, 1990). ${ }^{1}$

De las nociones anteriores, se excluyen las instiluciones estatales de carácter central, descentralizadas o autónomas y los partidos políticos. La sociedad civil incluye: i) Organizaciones dedicadas a la prestación de servicios a diversos sectores, territorios o comunidades, entre éstas se hallan instituciones de desarrollo, promoción, culturales, filantrópicas, de servicio y de emergencia; ii) Organizaciones prestatarias de servicios y de movilización social; ejemplos de ellas son: organizaciones de medio ambiente, género y derechos humanos; iii) Organizaciones representativas de intereses económicos y sociales de clases, grupos, gremios y comunidades. (González, 1991)²

Debido a las limitaciones de espacio y para deslacar los procesos y estructuras de las ONG'S no se harán análisis exhaustivos de aquellos organismos Iradicionales de la sociedad, los cuales existían antes del inicio de los conflictos políticos militares.

\section{Crisis centroamerlcana y socledad civli}

i-La década de los afios ochentas encuentra a los paises de Centro América involucrados en una crisis política y social, la cual tiene sus ralces afincadas en la historia acumulada de insatisfacciones económicas y de opresiones.para de los sectores populares de los paises centroamericanos. Los seculares desequilibrios de los procesos económicos, las reinvindicaciones frustradas y los derechos individuales o sociales sistemáticamenle violados por acciones u omisiones de los Estados centroamericanos, denotan la opción de las clases dominanles centroamericanas por los regímenes políticos diclatoriales y la renuncia del consenso como estilo de dominación, durante las décadas anteriores a la actual (Torres, 1989). ${ }^{3}$

Pero esta renuncia al dominio por medio del consenso y el subsecuenle debilitamienlo de los órganos de la sociedad civil, es un proceso asociado a la historia de las naciones centroamericanas, quizás desde sus origenes. Si bien, las estructuras culturales y politicas que permiten la reproducción de la dominación de unos grupos sociales sobre el resto de la sociedad en un período dado, se encuentran vinculadas como un todo integrado, en cualquier país, inclusive en Centroamérica, el caso del Islmo, es el de la menor aportación de la sociedad civil en la lunción de la dominación. 
Para comprender la naturaleza de las funciones de la dominación por los órganos de la sociedad civil, abundaremos en los siguienles conceptos.

"La sociedad civil está formada por el conjunto de los organismos vulgarmente llamados privados.. y que corresponden a la función de hegemonía que el grupo dominante ejerce en toda la sociedad " (Portelli, Hugues, 1978).4

La sociedad civil en los países desarrollados es una totalidad compleja, la cual cubre un campo muy amplio, para dirigir a lodo el conjunto de la sociedad en un periodo histórico dado y en función de los dilerenles estratos y grupos sociales y por ello, puede ser considerada bajo los siguientes aspectos: a) como ideologia de las clases dirigentes, abarcando diferentes ramas culturales o ideológicas como el arte, la ciencia, las leorías económicas, el derecho, etc; b) como concepción de mundo permeabilizada en los diferentes estratos sociales, a los cuales vincula a las clases dirigentes, a través de la filosolía, religión, sentido común y folklore; c) como eslructuras u organizaciones, creadoras y dilusoras de contenidos e instrumentos ideológicos por medio de sistemas escolares, medios de comunicación, bibliolecas, etc. (Portelli, 1978). ${ }^{5}$

En la realidad social de Centroamérica, las ideologías, las concepciones de mundo y las organizaciones creadoras y difusoras de ideología han sido raquíticas y su cobertura tradicionalmente se ha destinado a las élites e ínfimas capas medias, siendo muy difícil la permeabilización hacia los sectores populares, los cuales en la mayoría de paises de la región, muestran niveles elevados de analfabetismo y exclusión de los procesos cullurales dominantes.

Aparte de esto, los contenidos ideológicos difundidos en la región, revelan poca originalidad en su generación o creación, por lo cual, la pertinencia, coherencia y eficacia de sus efectos formadores de opinión y de actitudes sociopolíticas, mostraron su incompetencia ante los primeros movimientos reinvidicativos, solidarios y de oposición política, observados a partir de la década de los ańos setentas.

El campo de la sociedad civil es muy vasio y en él sólo las ideologías orgánicas, es decir, aquellas vinculadas a los grupos o clases fundamentales de la aclividad económica y sociopolítica, resultan importantes para el análisis de la realidad social. En la mayor parte de paises de CenIroamérica, las necesidades de reproducciones ideológicas por parle de las clases dominantes, no conllevaron en décadas pasadas, a la creación de un bloque coherente de intelectuales especializados en dilerentes quehaceres culturales. Por ello, no hubo profusidad en las labores 
intelectuales, en la producción e impacto en los diferentes grupos sociales; lo cual se traduce en desmedro de las formas más sofislicadas como la ciencia y la filosofía y si más significativo en la formación del "sentido común y el folklore.

Una de las caracleristicas esenciales de la sociedad civil es su cohesión interna, independiente de los diferentes quehaceres y Iracciones culturales, en lo tocanle a la formación de la opinión pública. Así las iglesias, las escuelas, las universidades, los medios de prensa, los clubes $u$ organizaciones de beneficencia, consliluyen las instituciones principales de la formación de la opinión pública (Portelli, 1978). ${ }^{6}$

En Centroamérica, esa cohesión interna de la sociedad civil, empezó a mostrar fisuras desde la década de los sesentas, cuando algunos órganos como las iglesias denuncian acciones represivas del Eslado en contra de sectores populares u oposilores a los regímenes políticos y además participando en alternativas de ideologias políticas no aceptadas por las clases dominantes criollas.

Así mismo, las escuelas y fundamentalmente las universidades, evidenciaron un cultivo de filosolias y ciencias opuestas a la cultura dominanle. Los últimos ańos de la década de los sesentas, especialmente 1968, se constiluyó en escenario temporal, en el cual se rasgaron los velos de visiones de mundo falsas y conformantes de un quielismo social.

Los movimientos obreros, campesinos, de estudiantes y maestros, entre los más deslacados, patenlizaron la pérdida del conlrol de la dirección cullural de la sociedad por parte de las clases tradicionalmente dominantes en Centroamérica. La respuesta del Estado fue fundamentalmente represiva, es decir como sociedad políica ejerció su función de dominio directo a través de acciones del "aparalo coercitivo para intentar conformar a las masas del pueblo de acuerdo al lipo de producción, de economía y de sociedad concebido por las clases dominantes en ese momento.

La respuestas coactivas de los Eslados centroamericanos, Iortalecieron aun más a la sociedad política, es decir, a la organización burocrática militar, la cual asumió como funciones principales, las siguientes: 1a.) El control de los grupos sociales disidentes con la dirección de la clase fundamental dominante en las relaciones económicosociales y políticocullurales; para el desarrollo de esta función se uliliza la coerción con mayor o menor legalidad. 2a) Las situaciones de uso mayor de coacción, en siluaciones de excepción legal como medio básico de ejercer la dominación y ante la incompetencia de la sociedad civil, para mantener la 
dirección de las acciones de los grupos sociales subalternos.

Esla "división del trabajo cultural y coaclivo", asumido por la sociedad civil y la sociedad política, respeclivamente, no significó o signitica una separación e incomunicación entre ambas. Por el contrario, hay una colaboración estrecha entre las mismas.

Se trala de funciones que guardan correspondencia. Así, las recurrenles crisis sociales y políticas hacen a los burócratas y políticos de los aparalos de eslado, difundir discursos con visiones y expectativas sociales y políticas, función no usual u ordinaria dentro de la concepción liberal o neoliberal de Estado. Asi mismo, "cuando el aparalo de estado se muestra impolente para controlar una crisis orgánica, la clase dirigente. puede suscitar en el seno de la sociedad civil, organizaciones paramilitares que una vez consumada su misión se inlegrarán al Estado". (Portelli, 1978).?

Durante los años setentas se sigue consolidando la unidad práclica y empleo alternativo de las funciones ideológicas que procuran consenso y las lunciones de coerción. La dominación fundamentalmente por la fuerza aunque provisoria en olros paises, en la mayoria de paises centroamericanos se vuelve recurrenle y casi permanente en algunos de ellos y esto constituye indicio de la crisis de la organización política y social, cuando la clase dominante no posee la dirección ideológica, se impone por la fuerza (Portelli, 1978).

En eslas condiciones, se desarrollan durante los afios setentas y ochentas, movimientos sociales y políticos de carácter revolucionario que terminan por modificar los escenarios de Centroamérica en los años ochentas y en la década presenle.

Una breve caracterización de los escenarios políticos y sociales en los dilerentes países, durante esla década y la anterior, permite anolar los aspectos siguientes. Nicaragua, en los anos ochentas, experimenta un esfuerzo de reconstitución de la sociedad civil y de la sociedad políti$\mathrm{ca}$, tras el descalabro de la dinastía somocisla. Pero este esfuerzo resulla particularmente difícil en lo concerniente a consolidar la dirección cullural, ideológica y moral de la sociedad nicaragüense, tanto por la crisis ecómicosocial y política interna como por los condicionamientos y las limitaciones inducidas desde el exterior.

En Gualemala, los grupos gobernanles carecen de voluntad reformisla y de diálogo para afrontar los problemas sociales y bajo esta concepción, la menor concesión a los sectores populares es considerado como derrola por la clase dominante (Torres, 1989). ${ }^{\bullet}$ 
En Honduras a inicios de los setentas, se presentaba un movimiento campesino de mucha movilización social, reinvindicando tierras aunque generalmente no se trataba de propiedades privadas. Los intentos de estabilización se frustraron en 1972, cuando el gobierno civil bi-partidista fue derribado por el golpe militar de Oswaldo López Arellano, consolidando a los militares como gobernantes en espacios reformistas de corto plazo (Torres, 1989). ${ }^{10}$

En El Salvador, la experiencia de la guerra civil y sus coslos sociales elevados, lortalecieron aun más, durante los ochentas, a los aparatos militares como aspecto dominante de la sociedad política. El fin de la guerra y el desmontaje relativo del ejército, siginfica una crisis para la sociedad política constituída sobre las bases de la décadas anteriores.

Para la década de los años setentas, "debe reconocerse, en el conjunto de la región y con la excepción de Cosla Rica, que las fuerzas políticas moderadas no tuvieron ninguna oportunidad real para ser reconocidas como allernativa trente a gobiernos autoritarios y abiertamente respaldados por Estados Unidos."11

En la mayor parte de paises centroamericanos se formaron gobiernos militares autoritarios que atrontaron las luchas de las clases suballemas con mecanismos de fuerza y la colaboración de órganos privados formadores de opinión pública. Los regímenes políticos oscilarian de ejércitos prelorianos gobernanles a pretorianos árbilros (Echeverria, 1990). ${ }^{12}$

El desarrollo de las luchas de las clases subalternas fue creando condiciones para el surgimiento de organizaciones privadas de tipo reinvindicativo y solidario, las cuales en la medida en que fueron perseguidas y reprimidas, se fueron asociando más estrechamente a los movimienlos insurgentes de Centroamérica. En la medida en la cual, las contiendas se agudizan o exacerban, las organizaciones gremiales, sindicales, movimientos campesinos, cooperalivos y demás organizaciones de sectores Irabajadores y populares, son consideradas en el esquema bipolar como aliados o bases de apoyo de las clases subalternas organizadas en movimientos polílicos, partidos o guerrillas y bajo esa perspecliva son perseguidos y combatidos.

Esta interprelación de las fuerzas politicas y sociales se hace desde la perspectiva de la lucha contrainsurgente, tan en boga en los anos setentas y ochentas. Bajo esta visión del mundo social y político, las terceras fuerzas no cabian en ese esquema y por ende las organizaciones privadas tradicionales de la sociedad civil tendian a lormar opiniones favorables y juslificativas de los esquemas reduccionistas y de carácler coactivo. En muchos casos los silencios, especialmente de los medios 
de prensa, contribuian a la desinformación y la deformación de opiniones públicas.

En estas condiciones de bipolarismos y de bloques políticos, los resquicios de organización permitidos se filtraban en organizaciones de la sociedad civil tradicional, provistas de un nuevo sentido o bien, surgieron nuevos temas o reinvindicaciones lales como: el humanitarismo, la ayuda solidaria a desplazados de los cońliclos, las luchas ecológicas, inslituciones de ayuda a personas pobres o menesterosas, reinvindicaciones de género y demás campos de acción que para la decodilicación e interpretación de los actores principales de la sociedad polílica dominante, resultasen relativamente inócuos o de menor sospecha de colaboracionismo con los movimienlos políticos opositores.

En suma, las organizaciones de la sociedad civil de los sectores dominantes ceden, lo que es su primogenitura en otras sociedades, a lavor de la sociedad polílica, la cual con sus funciones coactivas detienen y apuntalan el derruido orden social de Centroamérica. Las organizaciones de la sociedad civil (sindicalos, cooperativas, movimienlos de masas, etc) de las clases subalternas son practicamente conminadas a vincularse a la sociedad politica emergente y a desarrollar sus funciones en condiciones de ocultamiento, lo cual resulta contradictorio a sus funciones esenciales de conducción y cohesion de intereses sociales similares. En eslas circunslancias, se explica la relativa recesión de la actividad gremial, solidaria y política de muchos movimientos sociales en Centroamérica en los ańos ochentas y la mayor reducción de la sociedad civil en paises con largos confliclos político militares.

En tales condiciones, se experimenló un vacio de organizaciones privadas o no gubernamentales, tanto de los sectores sociales dominanles como de las clases subalternas que atendieran, cohesionaran y condujeran a los sectores populares, especialmente a los grupos sociales en condiciones de pobreza, afeclados por los cofliclos y sectores de población socialmenle vulnerables como las mujeres, nif́os, etc.

Aunque las primeras organizaciones no gubernamentales (ONG'S) de ayuda humanitaria, se remontan a las décadas de los anos sesentas. La mayor parte de ONG'S surgieron y se desarrollaron en los anos ochenlas o noventas; en muchos casos en el marco de las contiendas bipolares. Cierlas organizaciones de asistencia humanitaria a población menesterosa, las cuales operaron en los anos ochentas y noventas fueron una continuación de las labores iniciadas en décadas anteriores, las cuáles desde una inspiración más racional y humanilaria comprendieron la necesidad de orientación para las personas marginadas del sistema económicosocial y cullural. 
Fue así como parte de la inspiración cristiana del Concilio Vaticano II y a inicios de los ańos sesentas, surgieron de la Iglesia Calólica, organizaciones de asistencia para la población pobre, bajo el nombre de CARITAS, las cuales desarrollaron lunciones de recepción de ayuda alimentaria de instituciones o personas, tanlo a nivel nacional o internacional, para luego distribuir entre lamilias necesiladas. En muchas ocasiones se donaba ropa y medicina a familias pobres.

En la década de los ochenlas, esle tipo de instituciones que trabajan con ayuda alimentaria serán forlalecidas con fondos procedentes Naciones Unidas, Europa y Norteamérica. Según el Informe de la Comisión Kissinger, los Estados Unidos aportan a la región, unos 100 millones de dólares anuales en promedio, en conceplo de ayuda alimentaria y a través del programa PL-480. Esta ayuda, según el mencionado informe, pretende ser un alivio a la siluación de la desnutrición en la región (Comisión Kissinger, 1984). ${ }^{13}$

Actualmente, el programa norteamericano de ayuda alimentaria es administrado por la Agencia Internacional de Desarrollo de los Estados Unidos (AID), la cual funciona conjuntamente con el Servicio Católico de Socorro (CRS), para canalizar los alimenlos a las organizaciones de CARITAS de cada pais de Cenlroamérica.

Las diferentes y encontradas interpretaciones de las causas de la crisis económica económica, social y política de los paises centroamericanos, elaboradas por distintos analistas o intelectuales de la sociedad civil centroamericana no coinciden en sus diagnóslico y mucho menos en las soluciones a los problemas de la vida social de la región. Ante ello y para dar cohesión a las percepciones de las sociedades civiles y políticas, la Comisión Kissinger, considera que Centroamérica tiene para finales de los años ochentas, necesidades financieras netas de 24 mil millones de dólares, para poder superar el deficit de la balanza de pagos e incrementar sus reservas; pero esa necesidad de londos y su empleo productivo, requiere del cumplimiento de las siguientes condiciones: reducción de los niveles de violencia, mejorar política económica, mejorar el ambiente económico global (reducción de inflación), disminución de los factores que amenazan la seguridad nacional norteamericana (política de contra-insurgencia) y promoción del desarrollo humano (Comisión Kissinger, 1984). ${ }^{14}$

La percepción de los problemas y soluciones hechas por la Comisión Kissinger, servirán de marco conceplual de referencia para el desarrollo de acciones futuras de las organizaciones estatales o privadas de la región, relacionadas con los problemas sefialados en el informe cilado. 
La orientación ideologica del inlorme Kissinger, refleja la preocupación por la reproducción del orden económico, político y cultural, para lo cual resulta particularmente importanle, la promoción del desarrollo humano, a través del logro de los siguientes objetivos: 10.) Disminución de la desnutrición, 20.) Mejoramienlo en los niveles educativos y 30.) En materia de salud, se pretende el acceso universal a servicios de atención primaria y el control del crecimiento demográfico.

Para lograr eslos objelivos se recomienda aumentar los suministros concedidos por el programa PL-480, capacitar y mejorar a las instituciones regionales que administran la ayuda alimenlaria. También se sugiere mejorar la educación básica, desarrollar la formación lécnica o vocacional y el fortalecimiento de las universidades; lo cual se concretizaría con una nueva affabetización, reforzamienlo de centros de ensenanza técnica como el Institulo Cenlroamericano de Administración de Empresas (INCAE), concesión de becas, etc. En la medida de lo posible la ejecución de estas metas se hará a través de programas de asistencia técnica apoyados por la AID y preferentemente por medio de contrapartes nacionales formadas por organizaciones voluntarias privadas (ONG'S).

Además de eslas recomendaciones de desarrollo humano, y de restauración de los requisitos básicos para el relanzamienlo de la cultura dominante, el Informe Kissinger propone a los Estados Unidos, olorgar a El Salvador y a Honduras mayores niveles de ayuda militar. Recomendando específicamente para Honduras una mayor dotación para asumir el papel de gendarme regional: "... a fin de conseguir un poder confiable de disuación y de enlrentar renovados esluerzos para crear movimientos subversivos" (Comision Kissinger, 1984). ${ }^{15}$

De las relerencias anteriores, se deriva la preocupación norteamericana por la reslauración de la sociedad civil y la mayor consolidación de la sociedad política (ejércilos), ante las luchas de las clases suballernas. Todas estas preocupaciones y estuerzos no son expontáneos y graluito, ya que responden a la concepción geopolítica de seguridad regional norteamericana.

La dualidad de la política norleamericana para la región de Centroamérica, a tinales de la década de los ochentas, impone serias restricciones para el desarrollo y consolidación de la sociedad civil. Las funciones de las ONG'S, se limilan a lo lolerado por los regímenes militares de la región, lo cual, restringe las acciones e impacto de los programas de ayuda o desarrollo humano que las ONG'S impulsan.

Por otro lado, la restauración y consolidación ideológica de la sociedad civil, quedará en suspenso hasla el logro de la pacificación de 
Centroamérica y sólo a través de procesos de diálogo y negociación polílica, los cuales de suyo constiluyan la base sobre la que se construyan los nuevos consensos nacionales.

Los países de Centroamérica presentan diferencias importantes en términos sociales y políticos como anteriormente se ha sefialado, no obstante, el denominador común es la siluación global de subdesarrollo y de crisis con diferentes gradaciones. En tal sentido, los programas a ayuda internacional, dirigidos a intituciones eslatales y organizaciones no gubernamentales van dirigidos a las distintas facetas del subdesarrollo y pobreza endémica de la región; sus campos priorilarios de acción son: ayuda de emergencia a relugiados, desplazados, etc., asislencia alimenraria, reactivación económica y desarrollo social.

La ayuda alimentaria han tenido una gran relevancia, tanto por sus consecuencias miligantes para el problema del hambre como por su impacto político en las poblaciones a las que se suministra dicha ayuda. La principal agencia en la región es el Programa Mundial de Alimentos (PMA), que olorga recursos a programas gubernamentales, según la capacidad operativa de las agencias estatales (Gars1, 1992). ${ }^{16}$

Algunas de las poblaciones beneficiarias de los programas de alimenlos se consideran "clientela política" del personaje, partido o institución que distribuye la ayuda. Sin embargo, esta consecuencia o función latente se ha reducido con la modalidad de "alimenlos por trabajo", la cual exige a los beneficiarios, la realización de obras comunales que dignilican el trabajo y justilican la paga con alimentos; disminuyéndose así el "clientelismo".

La mayor parte de la ayuda alimentaria se distribuye por medio de instituciones gubernamentales y los programas de menor impacto son distribuidos por organismos internacionales no gubernamentales. Los programas y organizaciones más deslacados en esle rubro son: Programa Mundial de Alimentos y PL480 Título II (Public Law, Title II-Emergency and Privale Assislance Programs). Las organizaciones distribuidoras más significaliva son: CARE, Catholic Relief Services (CRS), CARITAS, CESAD y otras descritas en el cuadro número uno de Anexos.

En lo relerente a organizaciones de la sociedad civil en Centroamérica, según investigaciones recientes, el número aproximado de fundaciones y organismos afines de Centroamérica sumaba un lotal aproximado de 1331 instituciones esencialmente de naluraleza juridica privada (Fundación Arias, 1992) ${ }^{17}$

El estudio anteriormente citado, hace una clasilicación de fundaciones y organismos afines en base sus respeclivos campos de acción, 
distiguiéndose un poco más de treinta categorías, de las cuales las de mayor frecuencia son: grupos de población con el $15.7 \%$, asistencia económico-social (11.3\%), capacitación (10.2\%), desarrollo $(7.4 \%)$, salud, farmacodependencia, nutrición y rehabilitación $(8.7 \%)$. Las cinco categorias de campos de acción representan más de la milad de todas la organizaciones no gubernamenlales existenles en Centroamérica hasta 1992. La categoría grupos de población incluye campos especílicos como: discapacitados, etnias, lamilia, juventud, mujer, nifiez y tercera edad. Para una idea más completa de la distribución de trecuencias absolulas de las ONG'S de Centroamérica, clasificadas según campos de acción, véase Cuadro No. 2 de anexos.

Considerando el número de fundaciones y organismos afines por pais, resultan datos sumamente curiosos. EI Salvador es el pais con menores trecuencias de este tipo de crganizaciones. Nicaragua y Guatemala tienen proporciones similares y Honduras y Costa Rica destacan con las mayores trecuencias, tal como puede apreciarse en la tabla y gráfico correspondiente.

\section{Tabla 1}

Frecuenclas Absolutas y Relatlvas de Fundaciones y Organizaclones

Según Palses de Centroamérica, 1992.

\begin{tabular}{|l|c|c|}
\hline Pals & Frecuencia absoluta & Frecuencia relativa \\
\hline Gualemala & 212 & 15.9 \\
El Salvador & 169 & 12.7 \\
Honduras & 364 & 27.4 \\
Nicaragua & 208 & 15.6 \\
Costa Rica & 378 & 28.4 \\
\hline Tolal & 1331 & 100.0 \\
\hline
\end{tabular}

Fuente: Elaborado en base a datos de la Fundación Arias. " Guia de Fundaciones y Organismos Afines de Centroamérica, 1992. P.P: 497-501

Los datos anteriormente aludidos, sugieren una hipólesis explicativa de la generación de este tipo de organizaciones. Los paises centroamericanos con menores niveles de conlliclividad político-militar al interior de sus respeclivos terrilorios observan mayores frecuencias de organizaciones no gubernamentales (Casos de Costa Rica y Honduras). Por el 


\section{Gráfica N ${ }^{2} 1$ \\ Frecuenclas relallvas de fundaclones}

por palses de Centroamérlce, 1992

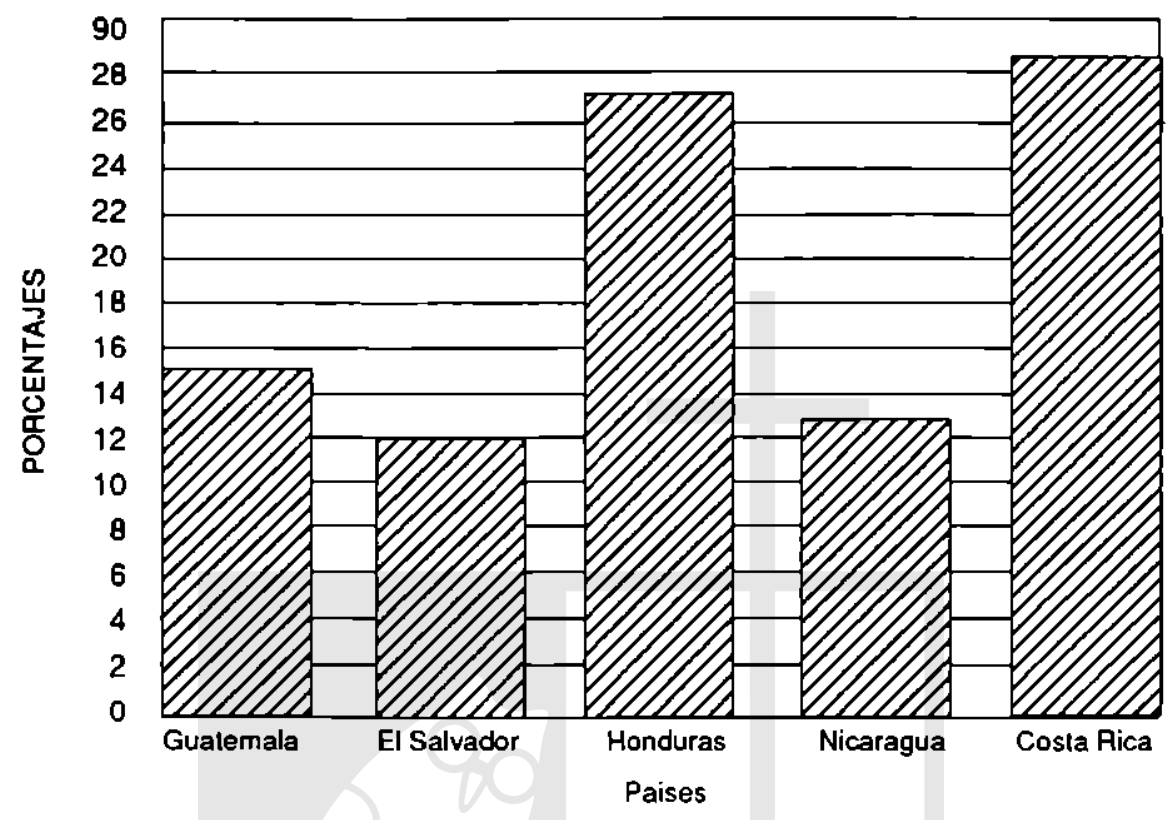

contrario, los paises con mayores niveles de conflictividad, al menos en la década pasada, reporlan menores volúmenes de organizaciones no gubernamentales (Casos de El Salvador, Nicaragua y Guatemala). Esta hipótesis resulia compalible con los datos cuantitativos y con las consideraciones cualitativas sefialadas como es: el cierre de los espacios de organización en siluaciones de crisis sociales manilestadas en las dimensiones político-militares.

Cosla Rica presenta un desarrollo mayor de las ONG'S, dirigidos a la asistencia económico-social y lécnica, al fomento de centros de capacitación, programas de desarrollo e investigación, alłabetización y de educación, formación en geslión cooperaliva y capacilación campesina.

Dadas las condiciones de distribución de la propiedad y del ingreso en Cosla Rica, resulta coherente el mayor desarrollo de la sociedad civil en este país. El resultado actual de las organizaciones no gubernamentales y la relativa consolidación de la sociedad civil también se explica por la acumulación histórica de experiencias y la reducción de los aparalos militares, sitemáticamente sostenida en este país. 
En Honduras, las organizaciones no gubernamentales más destacadas son las de capacitación, especialmente comunitaria y campesina. También son imporlantes las organizaciones de carácler asistencialista y de promoción de grupos sociales en situación de pobreza o vulnerabilidad. En mucha de estas organizaciones ha sido deslacado el papel de las iglesias, principalmente la Iglesia Católica, en la lucha por los espacios sociales de acción Irente a las restricciones castrenses.

En Nicaragua, el desarrollo de las ONG'S, en la década de los ochenlas, se vincula a instituciones religiosas, tanto nuevas como tradicionales (CARITAS, Menonitas, etc); originalmente todas ellas desarrollan un trabajo de asistencia inmediata a poblaciones afectadas por los conflictos 0 en situaciones de pobreza extrema. Sin embargo, actualmenle, empieza a pertilarse una tendencia al aumento instituciones dedicadas a la capacitación, organización sociocultural, desarrollo y formación de grupos de población (organizaciones leministas).

En Guatemala, la crisis económica y los conflictos étnicos y políticos, expresados de alguna manera en la creciente guerra entre las Fuerzas Armadas gubernamenlales y el Ejércilo Guerrillero de los Pobres (EGP), minimizan los espacios de acción y desarrollo de las ONG'S. Instituciones católicas y evangélicas ejecutan acciones fundamentalmente de asistencia y secundariamenle, de desarrollo nural comunitario con poblaciones campesinas e indígenas, pero todo ello de modo incipiente, debido a las persecuciones u operativos del ejércilo.

\section{Crisls de la socledad clvil y las nuevas organizaclones no guber- namentales en EI Salvador}

Según los dalos de fundaciones y organismos afines, EI Salvador registra el menor número de ONG'S. Eslo de acuerdo a la hipólesis formulada antes, resulta coherente, si se consideran los doce años de guerra civil y lo cual significó una reducción de los espacios sociales de los organismos privados de la sociedad civil, especialmente, de aquellos que atienden las necesidades, la cohesión y conducción de las clases suballernas.

El recuento de organizaciones, en base a los datos de la Fundación Arias, los mayores volúmenes de ONG'S, se registran en los campos de acción: grupos de población formados por asociaciones de mujeres, ninos y tercera edad (17.2\%); asistencia económica, social o técnica (11.8 $\%)$; capacilación (10.7\%); farmacodependencia, salud, nutrición y rehabilitación (11.2\%). El resto de instiluciones tienen menores frecuencias y son menos del $50 \%$ del total de ONG'S existentes en el pais. 
Actualmente, El Salvador, atronta una transición política a través de la desmilitarización de la sociedad, luego de la tirma de los acuerdos de paz en 1992. En esle marco de nuevas condiciones, se abren espacios para la operación de las ONG'S, las cuales crecerán cuantitativa y cualitativamente en los próximos años, asumiendo papeles de asistencia y desarrollo, en interacción con las comunidades, pero a la vez, exigiendo al Estado el cumplimienlo y garantia, lanto de de los derechos humanos individuales como de los derechos humanos económicos, sociales y cullurales (EI Salvador, Constitución, 1992). ${ }^{18}$

El fortalecimienlo de la sociedad civil y la creación de un consenso nacional, requiere de las lunciones de organismos orientadores de grupos de población y movimientos sociales en sus respectivos procesos de organización y participación social; por medio de ellos, se irá creando una sociedad más democrálica y participaliva.

Esla tesis, es producto histórico de la emergenle sociedad civil en su interacción con los grupos de población, en condiciones muchas veces hostiles, para el desarrollo de las ONG'S. Por ello, el estudio de las ONG'S debe aproximarse a su hisloria, es decir desde la década del conflicio político y militar. En este sentido, el surgimiento y fortalecimiento de las ONG'S ha respondido a los siguientes factores de reproducción. (González, Vicłor, 1991). ${ }^{19}$

a) Ayuda económica de los Estados Unidos a El Salvador, por Iransterencia de tondos, para el apoyo a la balanza de pagos, venta de alimentos y donaciones. En el programa de donación de alimentos, a Iravés del PL-480, se reforzó a: Catholic Relief Services e instiluciones gubernamentales. La ayuda económica dada por AID, permitia mantener relaciones con 68 organizaciones no gubernamentales, de la cuales el $54.4 \%$ financiaban sus operaciones con fondos de esa agencia. A finales de los ochentas, la Fundación para el Desarrollo Económico y Social (FUSADES), los programas de privatización y sectores empresariales, concentraban el $80 \%$ de la ayuda de AID. En el cuadro No.3 se detallan algunos de los destinos de la ayuda olorgada por AID.

b) Las Reformas a inicios los años ochentas, contribuyeron a la formación de asociaciones civiles, especialmente cooperativas de carácter agropecuario; fortaleciéndose el movimiento cooperativo, en dos grandes confederaciones: Confederación de Asociaciones Cooperativas de la Reforma Agraria (CONFRAS) y Confederación de Asociaciones Cooperativas de El Salvador (COACES). En estas condiciones surgió la Unión Nacional Obrera Campesina (UNOC), la cual congrega sectores cooperativistas y trabajadores (González, 1991). ${ }^{20}$ 
El desarrollo de este lipo de organizaciones, generó un efecto multiplicador de otras organizaciones no gubernamentales, las cuales prestaban servicios o ayuda al movimiento obrero y cooperativo de El Salvador

c) El empobrecimiento y los costos sociales del conflicto, generados por la crisis económica, explicable por la inflación, fuga de capitales, recesión, elc, elevaron el número de familias pobres e incrementándose asi, las "exporlaciones de población" a otros países y los movimienlos de población desplazada hacia los centros urbanos más importantes del país, especialmente los próximos a zonas confliclivas.

Las primeras organizaciones de asislencia a la población pobre o desplazada fueron las comunidades calólicas y luego se fueron sumando a este esfuerzo otras organizaciones religiosas. Posteriormente, surgen y se desarrollan organizaciones no gubernamentales especializadas en asistencia en salud, vivienda, capacitación, etc. Las respuestas del gobierno frente a estas iniciativas, fue la creación de la Comisión Nacional de Desplazados (CONADES). En esle periodo se iniciaron, en el país, las operaciones de la Cruz Roja Inlernacional. Por su parte, los desplazados crean el Comité Cristiano Pro Desplazados (CRIPDES) en 1984.

Por otro lado, los coslos sociales y polílicos del conilicio, a finales de los años ochentas, llevaron a un buen número de fuerzas sociales al inlenlo de reconstrucción de una sociedad civil, frente a la experiencia del "Debate Nacional", en 1988. Para este evento se convoco: a 19 organizaciones de la empresa privada, las cuales representaban el 18.6 $\%$ de los invitados; un lotal de 51 organizaciones profesionales, instituciones educativas, culturales y humanitarias que reprsentaban el $50 \%$ de los invitados y un tercer bloque, formado por organizaciones laborales y populares que representaban el $32.4 \%$ de los invitados (Montes, 1988). ${ }^{21}$

Uno de los objetivos del Debate Nacional fue la búsqueda del consenso para la paz y la solución política del conflicto; además de generar una opinión mayoritaria a favor este propósito, se hicieron sefhalamientos a la realidad social del pais, caracterizada como: "injuslicia estructural marginadora de la inmensa mayoria, que imposibilita la reproducción malerial y espiritual de la mayor parte de la población " (Montes, 1988). ${ }^{2}$

Los alcances del debate nacional lueron de tipo moral y no tuvieron un mayor impacto polílico inmediato, pero sus acuerdos sirvieron de base hislórica para el surgimiento de y consolidación ullerior de la sociedad civil, frente al Estado y las fuerzas políticas en contienda. Tanto las consecuencias etico-políticas como organizativas derivadas del Debate 
Nacional, han influido en los acuerdos de paz y en el desarrollo de la sociedad civil.

d) La Cooperación Internacional. Mientras los Estados Unidos enviaban ayuda económica y mililar para reforzar a los gobiernos salvadorefios, la cooperación inlernacional de otros Estados e instiluciones privadas internacionales, propiciaban el fortalecimiento de las organizaciones de la sociedad civil. Estas acciones produjeron un efecto indirecto de control del Estado, en términos de mejorar el respelo y garantía de los derechos humanos, a la vez que se promovia a nuevos actores sociales: las ONG'S.

Las principales fuentes de linanciamiento de las organizaciones no gubernamenlales de El Salvador, en los úllimos años, han sido organismos similares de otros paises: Europa, Eslados Unidos, Canadá, etc. Dichos fondos fueron orientados para la ayuda en sobrevivencia, asistencia humanitaria, repatriaciones y repoblamientos.

Al aproximarse al final de la guerra y posteriormente, con la firma de los acuerdos de paz entre el gobierno y el FMLN, se ha dado un crecimienlo cuanlilalivo y cualitativo de las organizaciones no gubernamentales; lo cual se traduce en un relorzamiento de la sociedad civil a través de las ONG'S.

En una muestra de aproximadamente 100 organizaciones no gubernamentales, se pudo constatar que aproximadamenle la tercera parte de las ONG'S iniciaron sus operaciones entre 1988 y 1991 . Muchas ONG's carecen de personalidad juridica, pero eso no impide el desarrollo de actividades y su potencialidad de crecimiento para 1993 y los años futuros.

El estatus legal de la personalidad jurídica no es determinante para la realización de operaciones de las organizaciones no gubernamenlales, pero su posesión es un requisito legal que facilita sus acciones y significa: el reconocimiento del eslado y de la sociedad de una personalidad jurídica, idenlificada con delerminados objetivos y formas de organización.

Según el estudio muestral, los tipos de personalidad juridica registrados por las ONG'S, eran los siguienles: Entidades privadas de Utilidad Pública (55.6\%); Sociedades Mercantiles (4.4\%); Cooperalivas (3.3\%); Misión Internacional (2.2\%); En Trámite (13.3\%); Instilución Eclesiástica (1.1\%) y No Determinado (20\%)(Ver Cuadro No.4). De los dalos, se concluye: la mayor parte de ONG'S están jurídicamente como corporaciones o lundaciones privadas de utilidad pública e inscrilas en los registros correspondientes del Ministerio del Interior. 
Por el lipo de organización, lo cual eslá asociado con objetivos y acciones desarrolladas por las ONG'S, la mayor parte de las mismas son calificadas como instituciones de promoción humana y desarrollo social $(61 \%)$; la mayor parte de estas organizaciones trabajan directamente con poblaciones específicas de sus respectivos proyectos. En segundo nivel de importancia, aparecen los Centros de Estudios, los cuales generalmente hacen investigaciones, consultoria, asesorias, elc. Los cuerpos coordinadores, son instancias de organización creados por cooperativas, asociaciones comunitarias $o$ de asistencia que integran un nivel superior de planificación y ejecución de esfuerzos entre organizaciones afines.

De acuerdo a las áreas de Irabajo, las ONGS, manifiestan 16 modalidades, las cuales van desde asislencia a nif́os, ancianos en situación de abandono o pobreza hasta organizaciones que desarrollan varios tipos de actividades en diferentes áreas y las cuales representan el $46.6 \%$ del tolal de ONGS. También deslacan, organizaciones con actividades de promoción social $(14.8 \%)$, esludios e invesligaciones (11.4\%). El resto de aclividades se lienen individualmente menor proporción y se refieren a las áreas de salud, asistencia de emergencia, producción económica, esludios etc, las cuales suman $27.2 \%$.

La mayororia de organizaciones no gubernamentales Irabajan directamente con lamilias en siluación de pobreza (52.8\%). Entre estas organizaciones se hallan, lanto entidades privadas de utilidad pública como organizaciones comunales. La mayoría de organizaciones que trabajan con personas pobres, son de promoción humana y desarrollo social; pero algunos centros de estudio han desarrollado investigaciones sobre la siluación de pobreza de las familias salvadoreñas.

La finalización del conflicto político-militar y la consolidación del proceso de paz ha provocado una serie de consecuencias en favor del fortalecimiento de la sociedad civil. En primer lugar, buena parte de la energía social involucrada en el conflicto, especialmente de intelectuales, buscan actualmente lormas de organización para orientar, movilizar 0 preslar servicios a sectores sociales tradicionalmente marginados u oprimidos. Asi mismo, la apertura de espacios sociales y politicos para la participación democrálica, estimulan la organización popular en diferentes sentidos: asociaciones de vecinos, sindicalos, cooperativas, asociaciones civicas, elc.

Por olro lado, la experiencia histórica de la guerra, la apertura inlormativa de los medios de comunicación y olros faclores, han permilido la toma de conciencia de sectores de las clases medias o altas, para la organización e impulso de acciones de asistencia o promoción humana en comunidades pobres. 
Todos estos esfuerzos conducen a una nueva dimensión social del pals con un relorzamiento de las organizaciones de la sociedad civil, las cuales asumen papeles más decisivos para el desarrollo social, la promoción humana y la prestación de servicios. Por ello, algunas reflexiones clasifican a las organizaciones no gubernamentales en dos tipos: instituciones de movilización social e instituciones de servicios técnicos.

Esta dicotomla de las organizaciones no gubernamentales, no niega la tendencia de muchas de ellas para desarrollar, lanlo acciones de movilización social como de servicios técnicos.

Pero el papel de las ONG'S no se reduce unicamente al contenido de la anterior tipologla, potencial o latentemente, desarrollan labores de fortalecimienlo de la sociedad civil, en un proceso de disminución del poder de los aparalos coactivos del Eslado (Ejércilo) y de la disminución relativa de otros órganos de la sociedad política (Partidos e instituciones estatales).

Por su diseminación en todo el tejido social, las ONG'S, desempenan una función importante en la consolidación intelectual y orgánica de un nuevo bloque histórico en el pais. El consenso predominante entre ellas, gira en torno a la democratización, la participación, la desmilitarización y en consecuencia el lortalecimiento de la sociedad civil. 


\section{ANEXOS}

Cuadro No.1

Organlzaciones que Distrlbuyen Ayuda Allmentarla

en Centroamérlca.

Organizaclón

\begin{tabular}{|l|l|}
\hline $\begin{array}{l}\text { Programa Mundial de Alimentos } \\
\text { (PMA) }\end{array}$ & $\begin{array}{l}\text { Programas de ayuda en cada país } \\
\text { de Centroamérica, para de despla- } \\
\text { zados y retugiados (Naciones Uni- } \\
\text { das). }\end{array}$ \\
\hline CARE & $\begin{array}{l}\text { Distribuye alimentos a Iravés de } \\
\text { agencias gubernamentales (Priva- } \\
\text { da Internacional). }\end{array}$ \\
\hline Calholic Relief Services (CRS) & $\begin{array}{l}\text { Agencia de desarrollo y ayuda, } \\
\text { dona alimentos a Guatemala y El } \\
\text { Salvador, a Iraves de CARITAS. }\end{array}$ \\
\hline $\begin{array}{l}\text { Comilé Evangélico Salvadoreño } \\
\text { de Servicio y Desarollo (CESAD) }\end{array}$ & $\begin{array}{l}\text { Donaciones alimentarias de AlD-El } \\
\text { Salvador, a población desplazada. }\end{array}$ \\
\hline Wort Share & $\begin{array}{l}\text { Agencia de AID con programas ur- } \\
\text { banos y de servicio social (Guate- } \\
\text { mala), a través de alimentos por } \\
\text { trabajo y pagos monetarios, res- } \\
\text { peclivarmente. }\end{array}$ \\
\hline Banco de Granos y & $\begin{array}{l}\text { Con fondos del Gobierno Cana- } \\
\text { diense y de productores agrícolas } \\
\text { privados de Canadá, reparte ayu- } \\
\text { da alimentaria a través de Comité } \\
\text { Central Menonita y Food the } \\
\text { Children. }\end{array}$ \\
\hline Comunidad Europea & $\begin{array}{l}\text { Donaciones de leche en polvo, ce- } \\
\text { reales y aceites; enviados a de } \\
\text { paises de Centroamérica, a través } \\
\text { de Euronaid, Cresthale, Oxtam, } \\
\text { Aiuti Mondiale, elc. }\end{array}$ \\
\hline
\end{tabular}

Fuente: Garst, Rachel. La Ayuda Alimenlaria al Istmo Centroamericano. CADESCA, 1992.

\section{Tipo de ayuda}

Programas de ayuda en cada país de Centroamerica, para de desplazados y refugiados (Naciones Uni-

Distribuye alimenlos a lraves de agencias gubernamentales (Privada Inernacional). dona alimenlos a Guatemala y $\mathrm{El}$ Salvador, a traves de CARITAS

Comilé Evangélico Salvadoreño AID-E Agencia de AID con programas urbanos y de servicio social (Guatemala), a través de alimenios por trabajo y pagos monetarios, res-

Con fondos del Gobierno Canadiense y de productores agrícolas privados de Canadá, reparte ayuda alimentaria a traves de Comite Central Menonita y Food the reales y aceites; enviados a de paises de Centroamérica, a través Aiuti Mondiale, elc. 


\section{Cuadro $N^{2} 2$}

\section{Fundaciones y organizaclones por pais y campo de acclón Centroamérica, 1992}

\begin{tabular}{|c|c|c|c|c|c|c|}
\hline Campos de Acción & Guatemala & El Salvador & Honduras & Nicaragua & Cosla Rica & Total \\
\hline $\begin{array}{l}\text { Agresión y Maltralo } \\
\text { Asistencia Económi- }\end{array}$ & 0 & 0 & 1 & 2 & 0 & 3 \\
\hline ca, Social y Técnica & 37 & 20 & 35 & 19 & 40 & 151 \\
\hline Autogestión & 2 & 6 & 10 & 3 & 1 & 22 \\
\hline Beneficencia & 1 & 0 & 1 & 0 & 4 & 6 \\
\hline Capacitación & 25 & 18 & 45 & 21 & 27 & 136 \\
\hline Comunicación & 2 & 0 & 5 & 5 & 4 & 16 \\
\hline Coordinación & 8 & 1 & 6 & 5 & 11 & 31 \\
\hline Desarrolto & 30 & 9 & 0 & 20 & 39 & 98 \\
\hline Educación & 17 & 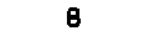 & 0 & 15 & 36 & 76 \\
\hline Emergencias & 2 & 2 & 0 & 2 & 2 & B \\
\hline \multicolumn{7}{|l|}{$\begin{array}{l}\text { Promoción de Em- } \\
\text { presas y Micro- }\end{array}$} \\
\hline empresas & 10 & 3 & 18 & 2 & 5 & 38 \\
\hline Grupos de Población & 21 & 29 & 84 & 22 & 53 & 209 \\
\hline Intraestructura & 0 & 1 & 3 & 5 & 0 & 9 \\
\hline Investigación & 5 & 3 & 10 & 11 & 35 & 64 \\
\hline Medio Ambiente & $\mathbf{3}$ & 2 & $B$ & 0 & 13 & 26 \\
\hline Organización & 1 & 5 & 11 & 9 & 3 & 29 \\
\hline Patrimonio & 0 & 0 & 1 & 2 & 3 & 6 \\
\hline Producción & 5 & 11 & 9 & 11 & 12 & 48 \\
\hline Promoción Educati- & 3 & 2 & 6 & 11 & 14 & 36 \\
\hline Promoción de la & & & & & & \\
\hline Paz y la Democracia & 1 & 0 & 1 & 3 & 4 & 9 \\
\hline Promoción Religiosa & 3 & 2 & 7 & 3 & 3 & 18 \\
\hline \multicolumn{7}{|l|}{$\begin{array}{l}\text { Salud, Nutrición, } \\
\text { Farmacodependencia }\end{array}$} \\
\hline y Rehabilitación & 14 & 19 & 40 & 16 & 27 & 116 \\
\hline $\begin{array}{l}\text { Sector Intormal } \\
\text { y Marginal }\end{array}$ & 4 & 8 & 16 & 4 & $\mathbf{8}$ & 40 \\
\hline Sector Rural & 7 & 5 & 20 & 4 & 10 & 46 \\
\hline \multicolumn{7}{|l|}{ Sector Turlstico } \\
\hline y U⿱bano & 2 & 0 & 6 & 2 & 1 & 11 \\
\hline Solidaridad & 1 & 0 & 3 & 1 & 5 & 10 \\
\hline \multicolumn{7}{|l|}{ Tecnologla } \\
\hline Apropiada & 1 & 6 & 3 & 0 & 10 & 20 \\
\hline Tradiciones & 0 & 0 & 0 & 3 & 2 & 5 \\
\hline Vletimes de Guerra & 0 & 3 & 1 & 4 & 2 & 10 \\
\hline Vivienda & 7 & 6 & 14 & 3 & 4 & 34 \\
\hline Total & 212 & 169 & 364 & 208 & 378 & 1.33 \\
\hline
\end{tabular}

Fuente: Elaborado en base a: Fundación Arias para la Paz y el Progreso Humano. "Gula de Fundaciones y Organismos Alines de Centroamérica ", 1992. 


\section{Cuadro No.3}

Donaclones de AID a ONG'S en EI Salvador

(En mlles de dólares)

\begin{tabular}{|c|c|c|c|}
\hline Organización & Año Fiscal 1985 & Año Fiscal 1986 & Año Fiscal 1987 \\
\hline \multicolumn{4}{|l|}{ Soporte a Sector Privado } \\
\hline $\begin{array}{l}\text { FEPADE } \\
\text { FUSADES } \\
\text { Empresarios Juveniles } \\
\text { IESC }\end{array}$ & $\begin{array}{r}0 \\
8,813 \\
300 \\
500\end{array}$ & $\begin{array}{r}0 \\
18,051 \\
650 \\
1,000\end{array}$ & $\begin{array}{r}6,000 \\
33,807 \\
3,000 \\
500\end{array}$ \\
\hline Sublotal & 9,613 & 19.701 & 43,307 \\
\hline Porcentaje del tolal & $35 \%$ & $66 \%$ & $80 \%$ \\
\hline \multicolumn{4}{|l|}{ Paciflcaclón } \\
\hline $\begin{array}{l}\text { Reforma Agraria } \\
\text { AIFLD } \\
\text { Tecnologla y servicio }\end{array}$ & $\begin{array}{r}2,000 \\
650\end{array}$ & $\begin{array}{l}2,500 \\
1,050\end{array}$ & $\begin{array}{l}2,500 \\
1,500\end{array}$ \\
\hline Subtotal & 2,650 & 3,550 & 4,000 \\
\hline Porcentaje del Total & $10 \%$ & $12 \%$ & $7 \%$ \\
\hline \multicolumn{4}{|l|}{ Desplazados } \\
\hline $\begin{array}{l}\text { CESAD } \\
\text { Caballeros de Malta } \\
\text { OEF Intemacional } \\
\text { Proyecto Hope } \\
\text { Wortd Relief }\end{array}$ & $\begin{array}{r}4.910 \\
0 \\
464 \\
2.645 \\
571\end{array}$ & $\begin{array}{r}3.101 \\
0 \\
0 \\
933 \\
442\end{array}$ & $\begin{array}{r}0 \\
500 \\
42 \\
1,100 \\
925\end{array}$ \\
\hline Subtotal & 8.950 & 4,476 & 2,567 \\
\hline Porcentaje del total & $31 \%$ & $15 \%$ & $5 \%$ \\
\hline \multicolumn{4}{|l|}{ Mlscelaneos } \\
\hline CENITEC & 0 & 0 & 600 \\
\hline Subtotal & 0 & 0 & 600 \\
\hline Porcentaje del total & 0 & 0 & $1 \%$ \\
\hline Mlscelaneos & Proyectos de & Servicio y & Desarrollo \\
\hline $\begin{array}{l}\text { CALMA } \\
\text { Sociedad Salesiana } \\
\text { Asociación Demográlica } \\
\text { Save the Children } \\
\text { Fundación Teletón }\end{array}$ & $\begin{array}{r}0 \\
0 \\
6.025 \\
600 \\
0\end{array}$ & $\begin{array}{r}220 \\
0 \\
2,000 \\
0 \\
0\end{array}$ & $\begin{array}{r}0 \\
1,100 \\
0 \\
1,300 \\
1,435\end{array}$ \\
\hline Subtotal & 6,625 & 2,220 & 3,835 \\
\hline Porcentaje del Total & $24 \%$ & $7 \%$ & $7 \%$ \\
\hline TOTAL & 27,478 & 29,947 & 54,309 \\
\hline
\end{tabular}

Fuente: AID El Salvador. Relerido por The Inter-Hemispheric Education Resource Center. "Private Organizations with U.S. Connections El Salvador". Mimeo. Pag.7 


\section{Cuadro No.4 \\ Frecuencla, Según Estatus Legal de las ONGS}

\begin{tabular}{|lc|}
\hline Estatus Legal de las ONGS & Frecuencia relativa \% \\
\hline Entidad Privada de Utilidad P. & 55.6 \\
Sociedad Mercantil & 4.4 \\
Cooperativa & 3.3 \\
Misión Internacional & 2.2 \\
En trámite & 13.3 \\
Instilución Eclesiástica & 1.1 \\
(Católica) & 20.0 \\
No determinado & 100.0 \\
\hline Total & \\
\hline
\end{tabular}

Fuente: Muestreo de ONGS.

\section{REFERENCIAS BIBLIOGRAFICAS}

1. Cuenca, Breny. "Crisis Nacional y ONGS". PREIS-Programa Regional de Investigación sobre El Salvador, mimeo, San Salvador, 1990.

2. González, Victor. " Las Organizaciones No Gubernamentales (ONGs): Nueva expresión de la sociedad civil salvadoreña. PREIS, San Salvador, 1991. Pag.20.

3. Torres Rivas, Edelberto. " Crisis del Poder en Centroamérica". EDUCA, Centroamérica, 1989. P: 71.

4. Portelli, Hugues. "Gramsci y el Bloque Hisiórico ". Editorial Siglo Veintiuno, México. 1978.

5. Ibid, Pag. 18

6. Ibid. P-P: 24-27.

7. Ibid. Pag. 30

8. Ibid. Pag. 30

9. Torres Rivas. Op. Cit. Página 72.

10. Ibid. Página: 77.

11. Ibid. Pag. 77

12. Echeverrla, Santiago. " La dernocralización del país como principio de solución a la crisis y al conillicto armado." Revista de Esludios Centroamericanos (ECA), 1990, 503. P-P: 729-745.

13. Comisión Kissinger. Informe de la Comisión Nacional Bipartita Sobre Centroamérica. Ed. Diana. México. 1984. P.P: 92-93.

14. Crr. Ibid. P-P: 84-144.

15. Ibid. Página 136.

16. Garts, Rachel. "La ayuda alimentaria al Istmo Centroamericano". CADES. CA. 1992. P-P: $138-142$. 
17. FUNDACION ARIAS PARA LA PAZ Y EL PROGRESO HUMANO. " Guía de Fundaciones y Organismos Alines de Centroamérica ", 1992.

18. La Constitución de la República de El Salvador, en su Art. 1, inciso 2o. dice: " En consecuencia, es obligación del Estado asegurar a los habitantes de la República, el goce de la libertad, la salud, la cultura, el bienestar económico y la justicia social."

19. Cir. González, Victor. "Las organizaciones no gubernamentales -ONG'S: Una nueva expresión de la sociedad civil salvadoreña." p-p: 55-68.

20. Ibid. Páginas: 56-57.

21. Montes, Segundo. " El Salvador 1988: Estructura de Clases y Comportamiento de las Fuerzas Sociales ". Departamento de Sociologla y Ciencias Pollticas. San Salvador, 1988. P-P:60-72.

22. Ibid. Pag. 68. 